\title{
BOUNDARY VALUE PROPERTIES CONNECTED WITH SOME IMPROPER DIRICHLET INTEGRALS
}

\section{EBBE THUE POULSEN}

\section{Introduction.}

In connection with boundary value problems for second order uniformly elliptic differential operators on a domain $\Omega$ several authors (notably Morrey [1] and Sobolev [3]) have studied spaces of functions for which the Dirichlet integral

$$
\int_{\Omega}|\nabla u|^{2} d x=\int_{\Omega} \Sigma\left|\frac{\partial u}{\partial x_{i}}\right|^{2} d x_{1} \ldots d x_{n}
$$

is finite. In particular, it is well known that such functions are locally square integrable and that they have square integrable boundary values (in the sense mentioned below) on smooth parts of the boundary $\partial \Omega$ of $\Omega$.

In the present paper we study the boundary behavior of functions for which a certain improper Dirichlet integral

$$
\int_{\Omega} \sum a_{i j}(x) \frac{\partial u}{\partial x_{j}} \frac{\partial \bar{u}}{\partial x_{i}} d x
$$

is finite. We assume that the quadratic form

$$
F(\xi, \bar{\xi})=\sum a_{i j}(x) \xi_{j} \bar{\xi}_{i}
$$

is positive definite in $\Omega$, but not necessarily uniformly. We shall see that the existence of boundary values depends on whether $F(\xi, \bar{\xi})$ becomes semidefinite on $\partial \Omega$, and if it does, it is also relevant how fast it degenerates as we approach the boundary. Most of our results have predecessors in Višik's paper [4], but we find our results more complete than his-for quadratic forms of sufficiently "uniform" behavior near the boundary our theorems furnish necessary and sufficient conditions for existence of boundary values.

The results of the present paper have been used in an investigation of problems connected with elliptic operators that degenerate on the boundary. We intend to publish the results in a later paper.

Received March 25, 1960. 


\section{Existence of boundary values.}

For simplicity, we shall restrict ourselves to the consideration of plane parts of the boundary, since the general case of smooth parts of the boundary can be derived from the plane case by transformations of the domain (all our theorems are of a local nature). Also, for simplicity, we assume that the coefficients $a_{i j}$ are continuous in $\Omega$.

It follows that the quadratic form $F(\xi, \bar{\xi})$ is uniformly positive definite on compact subsets $K$ of $\Omega$, that is, there exists a constant $\alpha=\alpha(K)>0$ such that

$$
F(\xi, \bar{\xi}) \geqq \alpha|\bar{\xi}|^{2}
$$

for all $x \in K$ and all complex vectors $\xi$.

If $u$ is a function whose derivatives (in the sense of distributions) are again functions, we shall denote its gradient by

$$
\nabla u=\left(\frac{\partial u}{\partial x_{1}}, \ldots, \frac{\partial u}{\partial x_{n}}\right)
$$

Let $\mathfrak{M}$ be the space of those functions for which

$$
\|\mid u\|^{2}=\int_{\Omega} F(\nabla u, \nabla \bar{u}) d x
$$

is finite. If $\Omega^{\prime}$ is a subdomain of $\Omega$ with compact closure in $\Omega$, then

$$
\int_{\Omega^{\prime}}|\nabla u|^{2} d x<\infty
$$

for all $u \in \mathfrak{M}$, and hence (see for instance Schwartz [2, p. 37])

$$
u \in \mathscr{L}^{2}\left(\Omega^{\prime}\right) \quad \text { for all } \quad u \in \mathfrak{M} \text {. }
$$

It is easily seen that $\mathfrak{M}$ is a Hilbert space under the norm

$$
\|u\|_{\mathfrak{M}}=\left(\|\| u\|\|^{2}+k\|u\|_{\mathscr{L}^{2}\left(\Omega^{\prime}\right)}^{2}\right)^{\frac{1}{2}}
$$

for $k>0, \Omega^{\prime} \neq \emptyset$, and that all norms of this form (for varying $k$ and $\Omega^{\prime}$ ) are equivalent.

Finally, note that it follows from the results of Morrey [1] that the identity mapping of $\mathfrak{M}$ into $\mathscr{L}^{2}\left(\Omega^{\prime}\right)$ is compact.

Let $\mathfrak{M}^{0}$ denote the closure in $\mathfrak{M}$ of the space $C_{0}^{\infty}$ of infinitely differentiable functions with compact support in $\Omega$.

As said above, we shall restrict ourselves to the consideration of plane boundary pieces which we will assume contained in the plane $\left\{x_{n}=0\right\}$. We shall use the following notations:

$\Gamma$ will denote a domain in $E^{n-1}$, and we shall define 
and

$$
\Gamma_{h}=\Gamma \times\left\{x_{n}=h\right\},
$$

$$
\Gamma_{h, k}=\Gamma \times\left\{h<x_{n} \leqq k\right\} \text {. }
$$

For convenience, we shall often denote the point $x=\left(x_{1}, \ldots, x_{n}\right) \in E^{n}$ by $x=\left(y, x_{n}\right)$, where we put $y=\left(x_{1}, \ldots, x_{n-1}\right)$.

For a function $u$ defined on $\Gamma_{h, k}$ we let $u_{t}$ denote the function on $\Gamma$ defined by

$$
u_{t}(y)=u(y, t), \quad y \in \Gamma, \quad h<t \leqq k .
$$

LEMMA 1. Let $\Gamma$ be a bounded domain in $E^{n-1}$, and assume that $\bar{\Gamma} \times\left\{0<x_{n} \leqq a\right\} \subset \Omega$. Then every $u \in \mathbb{M}$ can be changed on a set of measure 0 such that

1) $u_{h} \in \mathscr{L}^{2}(\Gamma)$ for all $u \in \mathfrak{M}$ and $0<h \leqq a$.

2) the mapping $h \rightarrow u_{h}$ is continuous from $\{0<h \leqq a\}$ into $\mathscr{L}^{2}(\Gamma)$ for all $u \in \mathfrak{M}$.

3) the mapping $u \rightarrow u_{h}$ is compact from $\mathfrak{M}$ into $\mathscr{L}^{2}(\Gamma)$ for all $h \in\{0<h \leqq a\}$.

Proof. The statements 1) and 3) are essentially contained in Morrey's theorems 7.1 and 8.5 in [1]. The proof of 2) is given below as part of the proof of theorem 2.

Theorem 2. Let $\Gamma$ be a bounded domain in $E^{n-1}$, and assume that

$$
\bar{\Gamma} \times\left\{0<x_{n} \leqq a\right\} \subset \Omega, \quad \Gamma_{0} \subset \partial \Omega .
$$

Assume also that there exists a function $\varphi(t)$, continuous and positive on the interval $\{0<t \leqq a\}$ with

such that

$$
\int_{0}^{a} \frac{d t}{\varphi(t)}<\infty
$$

$$
F(\xi, \bar{\xi}) \geqq \varphi\left(x_{n}\right)\left|\xi_{n}\right|^{2} \quad \text { for all } \quad x \in \Gamma_{0, a} .
$$

Then it is possible to define $u_{0} \in \mathscr{L}^{2}(\Gamma)$ in such a way that

1) the mapping $h \rightarrow u_{h}$ is continuous from $\{0 \leqq h \leqq a\}$ into $\mathscr{L}^{2}(\Gamma)$ for all $u \in \mathfrak{M}$.

2) the mapping $u \rightarrow u_{h}$ is compact from $\mathfrak{M}$ into $\mathscr{L}^{2}(\Gamma)$ for all $h \in\{0 \leqq h \leqq a\}$.

3) $u_{0}=0$ for all $u \in \mathfrak{M}^{0}$.

Proof. First note that if $\Omega^{\prime}$ is a subdomain of $\Omega$ with compact closure $\bar{\Omega}^{\prime} \subset \Omega$, then it follows by regularisation (see Schwartz [2, p. 21]) that all functions in $\mathfrak{M}$ can be approximated by infinitely differentiable functions with respect to the norm

$$
\left[\int_{\Omega^{\prime}}\left(F(\nabla u, \nabla \bar{u})+|u|^{2}\right) d x\right]^{\frac{1}{2}} .
$$


1) and 2). Now, let $v \in C^{\infty}(\Omega)$, and assume $0<t_{1}<t_{2} \leqq a$. Then we have

$$
\begin{aligned}
v\left(y, t_{2}\right)-v\left(y, t_{1}\right) & =\int_{t_{1}}^{t_{2}} \frac{\partial v}{\partial x_{n}}(y, t) d t \quad \text { for } \quad y \in \Gamma, \\
\left|v\left(y, t_{2}\right)-v\left(y, t_{1}\right)\right|^{2} & \leqq \int_{t_{1}}^{t_{2}} \frac{d t}{\varphi(t)} \int_{t_{1}}^{t_{2}} \varphi(t)\left|\frac{\partial v}{\partial x_{n}}\right|^{2} d t \\
& \leqq \int_{t_{1}}^{t_{2}} \frac{d t}{\varphi(t)} \int_{t_{1}}^{t_{2}} F(\nabla v, \nabla \bar{v}) d t,
\end{aligned}
$$

or, integrating with respect to $y$ :

$$
\left\|v_{t_{2}}-v_{t_{1}}\right\|_{\mathscr{L}^{2}(\Gamma)}^{2} \leqq \int_{t_{1}}^{t_{2}} \frac{d t}{\varphi(t)} \int_{\Gamma_{t_{1}, t_{2}}} F(\nabla v, \nabla \bar{v}) d x .
$$

It follows by continuity that this inequality holds for all $u \in \mathfrak{M}$, and a fortiori that

$$
\left\|u_{t_{2}}-u_{t_{1}}\right\|_{\mathscr{L}_{2(\Gamma)}}^{2} \leqq \int_{t_{1}}^{t_{2}} \frac{d t}{\varphi(t)}\|u\|_{\mathfrak{M}}^{2} \quad \text { for } \quad u \in \mathfrak{M} .
$$

If we denote the mapping $u \rightarrow u_{t}$ of $\mathfrak{M}$ into $\mathscr{L}^{2}(\Gamma)$ by $\Psi_{t}$, we see that

$$
\left\|\Psi_{t_{2}}-\Psi_{t_{1}}\right\|^{2} \leqq \int_{t_{1}}^{t_{2}} \frac{d t}{\varphi(t)} \quad \text { for } \quad 0<t_{1}<t_{2} \leqq a .
$$

Consequently $\Psi_{t}$ converges uniformly to a limit $\Psi_{0}$ for $t \rightarrow 0$, and since all $\Psi_{t}$ with $t>0$ are compact, the statements 1) and 2) follow.

As to 3 ), it is obvious that $u_{0}=0$ for all $u \in C_{0}^{\infty}$, and since $\Psi_{0}$ is continuous, we have $u_{0}=0$ for all $u$ in the closure $\mathfrak{M}^{0}$ of $C_{0}^{\infty}$.

When the assumptions of theorem 2 are fulfilled, we shall say that the function $u \in \mathfrak{M}$ has the boundary values $u_{0}$ on the part $\Gamma_{0}$ of the boundary. In particular, 3) says that all functions $u \in \mathfrak{M}^{0}$ have boundary values 0 on $\Gamma_{0}$. It is of obvious interest to know whether the converse holds: Do functions with boundary values 0 on the whole of $\partial \Omega$ belong to $\mathfrak{M}^{0}$ ?

That the answer is affirmative for domains with a smooth boundary in the case $F(\xi, \bar{\xi})=|\xi|^{2}$ is well known (Morrey [1]). His proof does not carry over to the situation treated here, but as we shall see, the theorem remains true under very natural assumptions on $F$. We shall only formulate and prove a local version of the theorem. 
Theorem 3. Let $\Gamma$ be a bounded domain in $E^{n-1}$, and assume that $\Gamma_{0, a} \subset \Omega$ and $\Gamma_{0} \subset \partial \Omega$. Assume further that there exists a function $\varphi(t)$, continuous and positive on the interval $\{0<t \leqq a\}$ with

such that

$$
\int_{0}^{a} \frac{d t}{\varphi(t)}<\infty
$$

and

$$
F(\xi, \bar{\xi}) \geqq \varphi\left(x_{n}\right)\left|\xi_{n}\right|^{2} \quad \text { for } \quad x \in \Gamma_{0, a}
$$

$$
a_{n n}(x) \leqq K \varphi\left(x_{n}\right) \quad \text { for } \quad x \in \Gamma_{\mathbf{0}, a} .
$$

Then we have $u \in \mathfrak{M}^{0}$ for all functions $u \in \mathfrak{M}$ with the properties

i) $u$ has boundary values 0 on $\Gamma_{0}$

ii) the support $S_{u}$ of $u$ is a compact subset of $\Omega \cup \Gamma_{\mathbf{0}}$.

Proof. We shall construct a sequence of functions $v_{k}=\omega_{k} u$ in $\mathfrak{M}^{0}$ such that $v_{k} \rightarrow u$ in $\mathfrak{M}$. The functions $\omega_{k}$ will be chosen to be of the form

$$
\omega_{k}(x)=\psi_{k}(\operatorname{dist}(x, \partial \Omega)),
$$

where $\psi_{k}(t)$ has the properties:

a) $\quad \psi_{k}(t)$ satisfies a Lipschitz condition on $0 \leqq t<\infty$;

b) $\psi_{k}(t)=0$ for $t \leqq \varepsilon_{k}$,

$0 \leqq \psi_{k}(t) \leqq 1 \quad$ for $\quad \varepsilon_{k} \leqq t \leqq k^{-1}$,

$\psi_{k}(t)=1$ for $k^{-1} \leqq t$.

Then $\omega_{k}$ satisfies a Lipschitz condition too, and since the function $u \in \mathfrak{M}$ is locally square integrable and has locally square integrable derivatives, the same is true of $\omega_{k} u$. By the assumptions on $\omega_{k}$ and $u$, however, $\omega_{k} u$ has compact support in $\Omega$, and it then follows by regularisation that $\omega_{k} u \in \mathfrak{M}^{0}$ for all $k$.

Now notice that since $u$ has compact support contained in $\Omega \cup \Gamma_{0}$, there exists a $k_{0}$ such that

$$
v_{k}(x)= \begin{cases}u(x) & \text { for } \quad x \in \Omega \sim \Gamma_{0, k^{-1}}, \\ \psi_{k}\left(x_{n}\right) u(x) & \text { for } \quad x \in \Gamma_{0, k^{-1}},\end{cases}
$$

when $k \geqq k_{0}$. For such $k$ we have

where

$$
\|\left.\left|u-v_{k}\right|\right|^{2}=\int_{\Gamma_{0, k^{-1}}} F\left(\nabla\left(1-\omega_{k}\right) u, \nabla\left(1-\omega_{k}\right) \bar{u}\right) d x=I_{k}{ }^{1}+I_{k}{ }^{2}+I_{k}{ }^{3},
$$

$$
I_{k}^{1}=\int_{\Gamma_{0, k^{-1}}}\left(1-\omega_{k}\right)^{2} F(\nabla u, \nabla \bar{u}) d x
$$




$$
\begin{aligned}
I_{k}^{2} & =\int_{\Gamma_{0, k^{-1}}}|u|^{2} F\left(\nabla\left(1-\omega_{k}\right), \nabla\left(1-\omega_{k}\right)\right) d x, \\
I_{k}{ }^{3} & =\int_{\Gamma_{0, k^{-1}}} 2 \operatorname{Re}\left(1-\omega_{k}\right) \bar{u} F\left(\nabla u, \nabla\left(1-\omega_{k}\right)\right) d x .
\end{aligned}
$$

Since $F(\xi, \bar{\xi})$ is positive definite, we have the Schwarz inequality:

$$
\left|F\left(\nabla u, \nabla\left(1-\omega_{k}\right)\right)\right|^{2} \leqq F(\nabla u, \nabla \bar{u}) \cdot F\left(\nabla\left(1-\omega_{k}\right), \nabla\left(1-\omega_{k}\right)\right) .
$$

Therefore,

$$
\begin{aligned}
2 \mid \operatorname{Re}(1 & \left.-\omega_{k}\right) \bar{u} F\left(\nabla u, \nabla\left(1-\omega_{k}\right)\right) \mid \\
& \leqq 2\left[\left(1-\omega_{k}\right)^{2} F(\nabla u, \nabla \bar{u})\right]^{\mathrm{k}}\left[|u|^{2} F\left(\nabla\left(1-\omega_{k}\right), \nabla\left(1-\omega_{k}\right)\right)\right]^{\mathrm{t}} \\
& \leqq\left(1-\omega_{k}\right)^{2} F(\nabla u, \nabla \bar{u})+|u|^{2} F\left(\nabla\left(1-\omega_{k}\right), \nabla\left(1-\omega_{k}\right)\right),
\end{aligned}
$$

so that

$$
\left|I_{k}^{3}\right| \leqq I_{k}^{1}+I_{k}^{2}
$$

Thus, the theorem will be proved when we establish the existence of a sequence of functions $\psi_{k}$ such that

and

$$
I_{k}^{1} \rightarrow 0 \text { for } k \rightarrow \infty
$$

Now, clearly

$$
I_{k}^{2} \rightarrow 0 \text { for } k \rightarrow \infty \text {. }
$$

while

$$
I_{k}^{1} \leqq \int_{\Gamma_{0, k-1}} F(\nabla u, \nabla \bar{u}) d x \rightarrow 0 \quad \text { for } \quad k \rightarrow \infty,
$$

$$
\begin{aligned}
I_{k}^{2} & =\int_{\Gamma_{0, k-1}}|u|^{2} a_{n n}(x)\left|\frac{d \psi_{k}}{d t}\left(x_{n}\right)\right|^{2} d x \\
& \leqq \int_{\Gamma_{0, k^{-1}}}|u|^{2} K \varphi\left(x_{n}\right)\left|\frac{d \psi_{k}}{d t}\left(x_{n}\right)\right|^{2} d x \\
& \leqq K \int_{0}^{k-1} \varphi(t)\left|\frac{d \psi_{k}}{d t}\right| \int_{\Gamma}^{2}\left|u_{t}\right|^{2} d y d t \\
& \leqq K\|u\|_{\mathfrak{M}}^{2} \int_{0}^{k-1} \varphi(t)\left|\frac{d \psi_{k}}{d t}\right|^{2} \int_{0}^{t} \frac{d \tau}{\varphi(\tau)} d t
\end{aligned}
$$

by the inequality $\left({ }^{*}\right)$ above (since $u_{0}=0$ ). Introducing the notation 


$$
\Phi(t)=\int_{0}^{t} \frac{d \tau}{\varphi(\tau)},
$$

our object is to make the integral

$$
\int_{0}^{k^{-1}} \varphi(t) \Phi(t)\left|\frac{d \psi_{k}}{d t}\right|^{2} d t
$$

small with the boundary conditions

$$
\psi_{k}\left(\varepsilon_{k}\right)=0, \quad \psi_{k}\left(k^{-1}\right)=1 .
$$

The minimizing function $\psi_{k}$ satisfies the equation

$$
\frac{d}{d t}\left(\varphi(t) \Phi(t) \frac{d \psi_{k}}{d t}\right)=0
$$

and hence we try $\psi_{k}$ of the form

$$
\psi_{k}(t)=C \log \Phi(t)+D,
$$

or

$$
\psi_{k}(t)=C \log \frac{\Phi(t)}{\Phi\left(k^{-1}\right)}+1 .
$$

For $C>0$ the equation $\psi_{k}(t)=0$ has exactly one root $\varepsilon_{k}$, and using the above expression for $\psi_{k}$ on the interval $\left\{\varepsilon_{k} \leqq t \leqq k^{-1}\right\}$ we get

since

$$
\int_{0}^{k-1} \varphi(t) \Phi(t)\left|\frac{d \psi_{k}}{d t}\right|^{2} d t=\int_{\varepsilon_{k}}^{k^{-1}} \frac{C^{2} d t}{\varphi(t) \Phi(t)}=C^{2} \log \frac{\Phi\left(k^{-1}\right)}{\Phi\left(\varepsilon_{k}\right)}=C
$$

$$
C \log \frac{\Phi\left(\varepsilon_{k}\right)}{\Phi\left(k^{-1}\right)}=-1 .
$$

Choosing $C=C(k)=k^{-1}$ the theorem follows.

The theorems 2 and 3 together show that if the quadratic form $F(\xi, \bar{\xi})$ does not degenerate too fast near the boundary piece in question, then the functions in $\mathfrak{M}$ have boundary values, and the functions that have boundary values 0 are essentially those in $\mathfrak{M}^{0}$.

\section{Non-existence of boundary values.}

We shall see next that if $F(\xi, \bar{\xi})$ degenerates too fast near $\partial \Omega$, then the functions in $\mathfrak{M}$ do not have boundary values in any reasonable sense, and, furthermore, the spaces $\mathfrak{M}$ and $\mathfrak{M}^{0}$ coincide if this degeneracy takes place along the whole of $\partial \Omega$. 
Theorem 4. Let $\Gamma \subset E^{n-1}$ be a bounded domain, and assume that

$$
\bar{\Gamma} \times\left\{0<x_{n .} \leqq a\right\} \subset \Omega ; \quad \Gamma_{0} \subset \partial \Omega .
$$

Assume further that there exists a function $\varphi(t)$, continuous and positive in the interval $\{0<t \leqq a\}$ with

such that

$$
\int_{0}^{a} \frac{d t}{\varphi(t)}=\infty
$$

and

$$
F(\xi, \bar{\xi}) \geqq \varphi\left(x_{n}\right)\left|\xi_{n}\right|^{2} \quad \text { for } \quad x \in \Gamma_{0, a}
$$

$$
a_{n n}(x) \leqq K \varphi\left(x_{n}\right) \quad \text { for } \quad x \in \Gamma_{0, a} .
$$

Then we have $u \in \mathfrak{M}^{0}$ for every function $u \in \mathfrak{M}$ which has compact support in $\Omega \cup \Gamma_{\mathbf{0}}$.

Proof. We construct a sequence $v_{k}=\omega_{k} u$ of functions in $\mathfrak{M}^{0}$ exactly as in the proof of theorem 3. For sufficiently large $k$ we have

where

$$
\begin{aligned}
\left\|u-v_{k}\right\|^{2} & =\int_{\Gamma_{0, k^{-1}}} F\left(\nabla\left(1-\psi_{k}\right) u, \nabla\left(1-\psi_{k}\right) \bar{u}\right) d x \\
& \leqq 2\left(I_{k}{ }^{1}+I_{k}{ }^{2}\right),
\end{aligned}
$$

and

$$
I_{k}^{1}=\int_{\Gamma_{0, k^{-1}}}\left(1-\psi_{k}\right)^{2} F(\nabla u, \nabla \bar{u}) d x \rightarrow 0 \quad \text { for } \quad k \rightarrow \infty
$$

Now, if we define

$$
I_{k}^{2}=\int_{\Gamma_{0, k^{-1}}}|u|^{2} F\left(\nabla\left(1-\psi_{k}\right), \nabla\left(1-\psi_{k}\right)\right) d x .
$$

$$
\Phi(t)=\int_{i}^{a} \frac{d \tau}{\varphi(\tau)}
$$

it follows from $\left(^{*}\right)$ in the proof of theorem 1 that

and since

$$
\left\|u_{a}-u_{t}\right\|_{\mathscr{L}^{2(\Gamma)}}^{2} \leqq \Phi(t)\left|\|u \mid\|^{2},\right.
$$

we have

$$
\left\|u_{a}\right\| \leqq C\|u\|_{\mathfrak{M}} \quad \text { for some } C
$$

when

$$
\left\|u_{t}\right\|_{\mathscr{L}^{2(\Gamma)}}^{2} \leqq C \Phi(t)\|u\|_{\mathfrak{M}}^{2} \quad \text { for some } C \text {, }
$$

$$
0<t \leqq \frac{1}{2} a \text {. }
$$

Thus, for sufficiently large $k \geqq 2 a^{-1}$ we have 


$$
I_{k}^{2}=\int_{0}^{k-1} \int_{\Gamma}|u|^{2} a_{n n}(x)\left|\frac{d \psi_{k}}{d t}\left(x_{n}\right)\right|^{2} d y d x_{n} \leqq K C\|u\|_{\mathfrak{M}}^{2} \int_{0}^{k^{-1}} \varphi(t) \Phi(t)\left|\frac{d \psi_{k}}{d t}\right|^{2} d t
$$

Choosing

$$
\psi_{k}(t)=1+k^{-1} \log \frac{\Phi\left(k^{-1}\right)}{\Phi(t)}, \quad \varepsilon_{k} \leqq t \leqq k^{-1},
$$

where $\varepsilon_{k}$ is determined by the relation

$$
\log \frac{\Phi\left(\varepsilon_{k}\right)}{\Phi\left(k^{-1}\right)}=k
$$

we get

$$
I_{k}^{2} \leqq K C\|u\|_{\mathfrak{M}}^{2} \cdot k^{-1} \rightarrow 0 \quad \text { for } \quad k \rightarrow \infty,
$$

and the proof is completed.

If we consider again the mappings $\Psi_{t}$ of $\mathfrak{M}$ into $\mathscr{L}^{2}(\Gamma)$, it is clear that

when

$$
\Psi_{t} u \rightarrow 0 \text { for } t \rightarrow 0
$$

$$
u \in C_{0}^{\infty} \subset \mathfrak{M}^{0} \text {. }
$$

On the other hand, if the coefficients $a_{i j}$ with $i, j \leqq n-1$ do not behave too badly, for instance if they are bounded, then it follows from theorem 4 that under the assumptions of that theorem there exist functions in $\mathfrak{M}^{0}$ for which

$$
\lim _{t \rightarrow 0} \Psi_{t} u \neq 0
$$

(choose, for instance, $v \in C_{0}^{\infty}(\Gamma)$, and define $u(x)=u(y, t)=v(y)$ for $0<t<\varepsilon$ and extend this $u$ to an infinitely differentiable function with compact support in $\Omega \cup \Gamma_{0}$ ). In this case, therefore, we have

$$
\lim _{t \rightarrow 0} \Psi_{t} u=0
$$

for all $u$ in the dense subset $C_{0}^{\infty}$ of $\mathfrak{M}^{0}$, but

$$
\lim _{t \rightarrow 0} \Psi_{t} u \neq 0
$$

for some elements of $\mathfrak{M}^{0}$. Consequently, by the principle of uniform boundedness, there are functions $u \in \mathfrak{M}^{0}$ for which $\Psi_{t} u$ does not have a limit for $t \rightarrow 0$. Thus, in this case it is impossible to ascribe boundary values to the functions in $\mathfrak{M}$.

\section{REFERENCES}

1. C. B. Morrey, Jr., Functions of several variables and absolute continuity, Duke Math. J. 6 (1940), 187-215. 
2. L. Schwartz, Théorie des distributions II (Act. Sci. Ind. 1122), Paris, 1951.

3. S. L. Sobolev, Nekotorye primeneniya funkcional'nogo analiza v matematičeskoi fizike, Leningrad, 1950.

4. M. I. Višk, Kraevye zadači dlya elliptičeskih uravnenit, vyroždayuščihsya na granice oblasti, Mat. Sb. N.S. 35 (77) (1954), 513-568.

\section{UNIVERSITY OF AARHUS, DENMARK}

